\title{
Comparative Studies on the Properties of Thyroglobulins from Various Animal Species
}

\author{
Tetsuya HOSHINO AND Nobuo UI \\ Department of Physical Biochemistry, Institute of Endocrinology, \\ Gunma University, Maebashi
}

\begin{abstract}
Synopsis
Thyroglobulins were extracted from the thyroid glands of one amphibia (Xenopus laevis), one aves (chicken) and five mammals (whale, rat, hog, monkey and human), and purified by means of salting-out with ammonium sulfate followed by DEAE-cellulose chromatography. Sedimentation coefficients, electrophoretic mobilities, isoelectric points, immunochemical properties as well as iodine contents and amino acid compositions of all or most of these thyroglobulins were determined and the results were compared with each other. In spite of a great variety of animal species studied, these thyroglobulins were found to be rather similar in various respects, while species specificity was clearly indicated. Most distinct differences were observed among amphibian, avian and mammalian thyroglobulins, especially in amino acid composition, isoelectric point and immunochemical properties.
\end{abstract}

Thyroglobulin, the major iodoprotein constituent of the thyroid, has been the subject of extensive studies in recent years. As a result, our knowledge on its nature and biological role has increased markedly. However, very little attention has been paid to thyroglobulins from species other than a few domestic animals or human, although the thyroid gland is present in all classes of vertebrates and is believed to play, in common, a physiologically important role as the site of iodine accumulation and thyroid hormone biosynthesis ( $c f$. Gorbman and Bern, 1962). Recent investigations on the ultracentrifugal composition of thyroidal iodoproteins and the iodination of these proteins in vivo (Lachiver et al., 1965 and 1966; Roche et al., 1968) employing the thyroid glands obtained from a variety of vertebrates have provided useful information, but no attempts have been made to compare the properties of thyroglobulin

Received for publication December 25, 1970. purified from these animals. Even in the case of a few mammals studied extensively, investigation from a comparative point of view is astonishingly lacking except some studies on amino acid composition (Rolland et al., 1966) and carbohydrate composition (Spiro and Spiro, 1965). It has been pointed out recently that the stability properties of newly-synthesized thyroglobulin from guinea pig, rat and beef are markedly different from each other (Schneider et al., 1970).

The present investigation was undertaken as an attempt to fill the gap in our current information concerning the nature of thyroglobulin. For this purpose, thyroglobulins were purified from two non-mammalian vertebrates (Xenopus laevis and chicken) and five mammals (whale, rat, hog, monkey and human), and comparisons were made with respect to chromatographic, ultracentrifugal, electrophoretic, immunochemical and other properties as well as iodine content and amino acid composition. 


\section{Materials and Methods}

\section{Thyroid Glands}

Thyroid glands obtained from two lower vertebrates and five mammals were used in the present investigation. Sources of animals are as follows.

Xenopus laevis: Approximately 300 adult male Xenopus laevis were kindly provided by Dr. Y. Hanaoka of the Department of Comparative Endocrinology of this Institute.

Chicken: Thyroid glands from each of approximately 300 male and female chickens were obtained at local chicken-yards.

Whale: Three frozen thyroid glands were kindly supplied by the Taiyō Fishery Co., Ltd., Tokyo. These glands were collected on the Antarctic Ocean and kept at a temperature of $-20^{\circ} \mathrm{C}$ or below until used.

Rat: A hundred Wistar rats (50 males and 50 females) weighing 150 to $200 \mathrm{~g}$ were sacrificed by cervic dislocation and the thyroid glands were separated immediately.

Hog: Approximately 40 hog thyroid glands were obtained at a local slaughter-house.

Monkey: Thyroid glands from 8 adult Rhesus monkeys were extracted with saline and crude thyroglobulin was prepared by salting-out with ammonium sulfate. This preparation was put at our disposal through the courtesy of Dr. Y. Kondo of this laboratory.

Human: Five normal human thyroids were obtained at autopsy.

\section{Preparation of Crude Extracts}

All the thyroid glands, except those of whale which had already been kept frozen, were at first freed from fat and extraneous tissues as far as possible and usually frozen. Frozen thyroid glands from whale, hog and human were sliced and extracted with three volumes of $0.9 \% \mathrm{NaCl}(\mathrm{pH} \mathrm{7)}$ for several hours at $2^{\circ} \mathrm{C}$, with occasional stirring (Ui and Tarutani, 1961). In the case of smaller animals, such as Xenopus laevis, chicken and rat, frozen or sometimes unfrozen glands were homogenized in a Waring blendor at $2^{\circ} \mathrm{C}$ with the same solvent. These extracts or homogenates were clarified by centrifugation for $15 \mathrm{~min}$ at 10,000 r.p.m. in the cold.

\section{Salting-Out}

Thyroglobulin was prepared from the crude extracts by salting-out with ammonium sulfate at approximately $2^{\circ} \mathrm{C}$ by the method previously reported (Ui and Tarutani, 1961). In most cases fractional precipitation between 1.50 and $1.80 \mathrm{M}$ ammonium sulfate was satisfactory, but a much higher concentration of ammonium sulfate was necessary to precipitate Xenopus laevis thyroglobulin, as will be described later.

\section{DEAE-Cellulose Chromatography}

Further purification and fractionation of thyroglobulin were carried out by DEAE-cellulose chromatography in a manner essentially similar to that described previously (Ui et al., 1961; Ui and Tarutani, 1961). For this purpose, each crude preparation of thyroglobulin prepared by salting-out was dialyzed against a phosphate buffer of $\mathrm{pH} 6.5$ at ionic strength 0.1 and applied to a column of DEAE- cellulose (Serva, $0.62 \mathrm{meq} . / \mathrm{g}$ ) equilibrated with the same buffer. The column size somewhat varied from experiment to experiment depending upon the amount of the protein to be applied, but the ratio of cellulose ionexchanger to protein was kept at approximately $60: 1$ on the dry weight basis. Stepwise elution was performed by increasing the ionic strength of the eluant by the addition of $\mathrm{NaCl}$ at a constant $\mathrm{pH}$ of 6.5. In the case of rat thyroglobulin, a little different elution schedule was adopted as described in the text.

Fractions eluted from the column were pooled and thyroglobulin was precipitated with ammonium sulfate usually after the solution was concentrated by negative-pressure dialysis. The wet precipitate collected by centrifugation was kept at $-5^{\circ} \mathrm{C}$ to $-10^{\circ} \mathrm{C}$ until used.

\section{Ultracentrifugal Analysis}

Sedimentation analysis was carried out in a Spinco model $\mathrm{E}$ ultracentrifuge equipped with a rotortemperature indicating and controlling unit. Runs were made at a constant temperature of around $20^{\circ} \mathrm{C}$ and at a rotor speed of 50,740 or 52,640 r.p.m. Prior to ultracentrifugation, the solution to be examined was dialyzed against a phosphate buffer of $\mathrm{pH} 6.5$ at ionic strength 0.2 , except in the case of crude extract where clarified solutions were subjected to centrifugal analysis after appropriate dilution with $0.9 \% \mathrm{NaCl}$. Sedimentation coefficients were calculated from schlieren patterns taken at $8 \mathrm{~min}$ intervals and the values obtained were corrected to a standard state corresponding to water at $20^{\circ} \mathrm{C}\left(\mathrm{s}^{\circ}{ }_{20, \mathrm{w}}\right)$ by a conventional manner.

\section{Polyacrylamide Gel Electrophoresis}

Electrophoresis was performed in a thin-layer of 4 $\%$ polyacrylamide gel (thickness: $1 \mathrm{~mm}$ ) according to a method essentially similar to that reported by Ogita (Ogita, 1965). A veronal buffer of pH 8.60 at ionic strength 0.10 was used as a solvent and electrophoresis was continued for $9 \mathrm{hr}$ at a constant current of $2 \mathrm{~mA}$ / $\mathrm{cm}$. 


\section{Determination of Isoelectric Point}

Isoelectric point was determined by the isoelectric focusing method (Svensson, 1962; Vesterberg and Svensson, 1966) at $5^{\circ} \mathrm{C}$ in an LKB 8101 column of 110 $\mathrm{ml}$-capacity as described elsewhere (Ui, 1971 a, b). Carrier ampholytes covering a $\mathrm{pH}$ range between 4 and 6 were used throughout this investigation.

\section{Immunological Methods}

Antisera against chicken, whale, hog or human thyroglobulin were prepared in rabbits by immunization with chromatographically purified antigen suspended in Freund's complete adjuvant. Usually each rabbit was injected subcutaneously with a total of approximately $10 \mathrm{mg}$ of thyroglobulin.

Precipitin tests by double diffusion in agar were made by the method of Ouchterlony ( $c f$. Ouchterlony, 1962) with $1 \%$ agar in $0.9 \% \mathrm{NaCl}$ of $\mathrm{pH} 7.0$.

\section{Chemical Analyses}

Iodine: Iodine was determined either by a modiffied method of Gross et al. (1948) or by using a Technicon Autoanalyzer.

Amino Acids: For the purpose of amino acid analysis, chromatographically purified preparations of thyroglobulin were thoroughly dialyzed against water and lyophilized. Approximately $2 \mathrm{mg}$ of each sample was hydrolyzed for $24 \mathrm{hr}$ at $110^{\circ} \mathrm{C}$ with $6 \mathrm{~N} \mathrm{HCl}$ in an evacuated, sealed tube. The hydrolyzate was analyzed with a Hitachi amino acid analyzer, model KLA-3B, by the method of Spackman et al. (1958). Corrections for the hydrolytic losses of threonine and serine were assumed to be $6 \%$ and $13.5 \%$, respectively, in view of the results (Hayashi and Iwai, unpublished) of amino acid analyses of non-iodinated hog thyroglobulin performed after various hydrolysis times. We are indebted to Prof. Iwai and his associates (Department of Protein Chemistry of this Institute) for the amino acid analyses.

\section{Results}

\section{Ultracentrifugal Analyses of Thyroid Ex- tracts and Crude Preparations Obtained by Salting-Out}

Thyroid extracts of several animal species were analyzed in the analytical ultracentrifuge. The sedimentation patterns obtained are shown in Figure 1. Only in the case of monkey, the pattern for the crude preparation of thyroglobulin prepared by salting-out with ammonium sulfate is recorded instead of that for the saline extract, because the latter prepa-
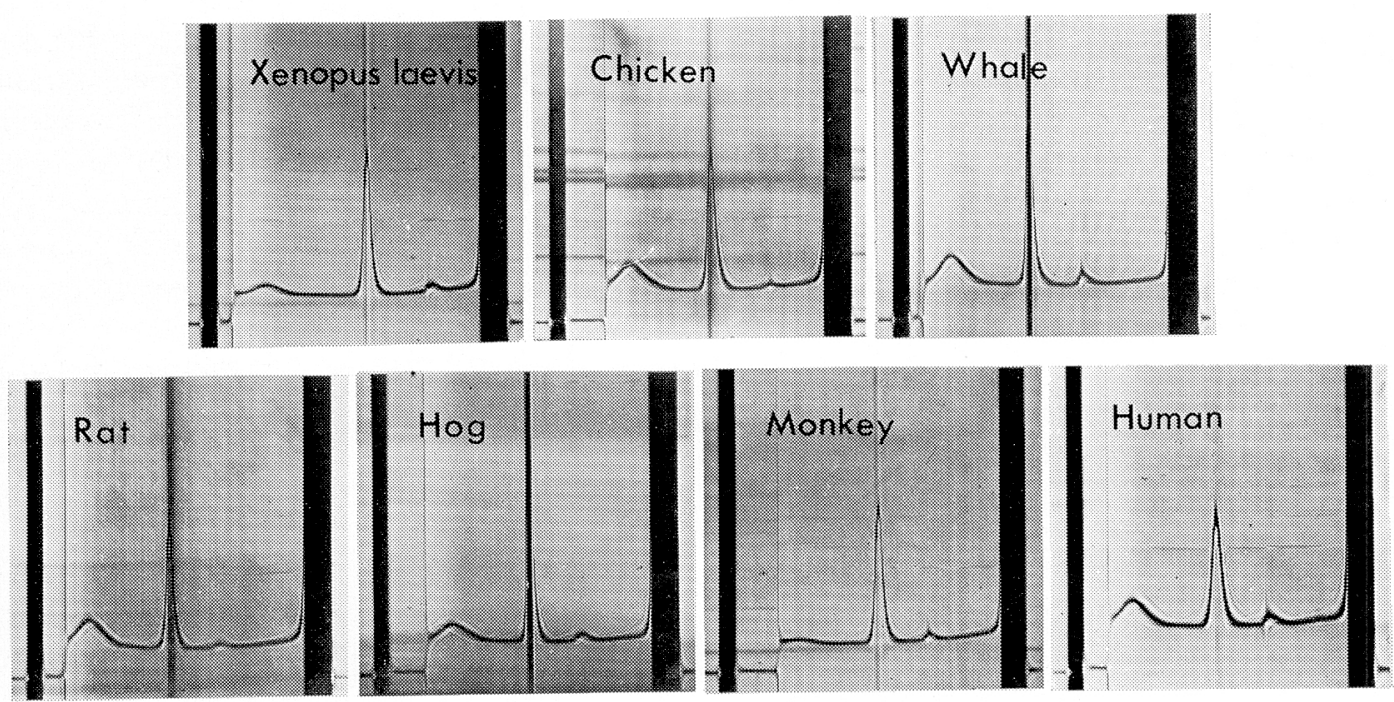

Fig. 1. Ultracentrifugal patterns of crude thyroid extracts obtained from various animal species. Only in the case of monkey, a crude preparation of thyroglobulin prepared by salting-out is recorded instead of thyroid extract. Total protein concentrations were nearly $1 \%$. These photographs were taken $25 \sim 33 \mathrm{~min}$ after the rotor speed reached a constant speed of 50,740 r.p.m. or 52,640 r.p.m. Sedimentation proceeds from left to right. 
ration was not measured ultracentrifugally.

It is evident from this figure that the composition of soluble macromolecular components in the thyroid is essentially similar in all the animals examined in this investigation. There can be seen at least three components in every case. Of them, the most predominant component is thyroglobulin having a sedimentation coefficient close to $19 \mathrm{~S}$. A minor component sedimenting faster than thyroglobulin corresponds to $27 \mathrm{~S}$ thyroid iodoprotein, which is closely related to thyroglobulin in various aspects (Salvatore et al., 1965). Another broad, asymmetric peak with the slowest sedimentation coefficient contains a mixture of cellular and blood proteins.

Although the presence of $12 \mathrm{~S}$ component, which is considered to be a stable subunit of thyroglobulin, has been reported in some nonmammalian vertebrates and occasionally even in mammals (Roche et al., 1968), no such component was found in the thyroid extracts of all the animal species studied.

Purification of thyroglobulin from the saline extracts was performed by salting-out with ammonium sulfate. Except in the case of Xenopus laevis, thyroglobulin was precipitated almost completely with ammonium sulfate between $1.50 \mathrm{M}$ and $1.80 \mathrm{M}$, in agreement with the observation with hog thyroid extracts previously reported (Ui and Tarutani, 1961). In some animals (whale and human), modified ammonium sulfate concentrations $(1.50 \mathrm{M}$ $1.90 \mathrm{M})$ were actually used, since slightly higher solubility was suspected. This point is, however, not certain, since no critical investigation was made on the solubility properties of these thyroglobulins.

On the other hand, Xenopus laevis thyroglobulin showed markedly higher solubility than other thyroglobulins; at $1.90 \mathrm{M}$ ammonium sulfate no appreciable precipitation occurred and the concentration of $2.6 \mathrm{M}$ had to be employed to obtain the precipitate in good yield.

Ultracentrifugal analysis on the crude preparations of thyroglobulin which were ob- tained by salting-out from various species indicated that the faster sedimenting component (27 S thyroid iodoprotein) had the same solubility in concentrated ammonium sulfate as $19 \mathrm{~S}$ thyroglobulin. The amount of slower component fairly decreased after salting-out but complete separation was not achieved. These results agree with the previous observation with hog thyroidal proteins (Ui and Tarutani, 1961).

\section{Chromatography on DEAE-Cellulose}

Previous investigations in this and other laboratories (Ui et al., 1961; Robbins, 1963; Shulman and Armenia, 1963; Valenta et al., 1968) have revealed that DEAE-cellulose chromatography is useful not only for the purification of hog, bovine or human thyroglobulin, but also for the purpose of fractionating these thyroglobulins into subfractions with varying iodine contents. On stepwise elution, fractions obtained at lower ionic strengths showed lower iodine content than those eluted at higher ionic strengths, and $27 \mathrm{~S}$ iodoprotein had a tendency to be eluted later. With these findings in mind, crude preparations of thyroglobulin from various animal species which were obtained by salting out were chromatographed on DEAE-cellulose and elution patterns were compared with each other. Stepwise elution curves obtained are given in Figure 2. In each experiment, the first peak not adsorbed at ionic strength 0.10 (except in the case of Xenopus laevis thyroglobulin as described below) and the last peak eluted at ionic strength 2.0 did not contain thyroglobulin and both were considered to be due to the contaminated materials.

In the case of hog (Fig. 2D), several peaks containing thyroglobulin were obtained on increasing ionic strength successively, indicating the marked heterogeneity of hog thyroglobulin. The main fraction was obtained at ionic strength 0.18 and showed a single boundary of $19 \mathrm{~S}$ on analytical ultracentrifugation. The fraction eluted at ionic strength 0.20 contained a trace amount of $27 \mathrm{~S}$ protein 

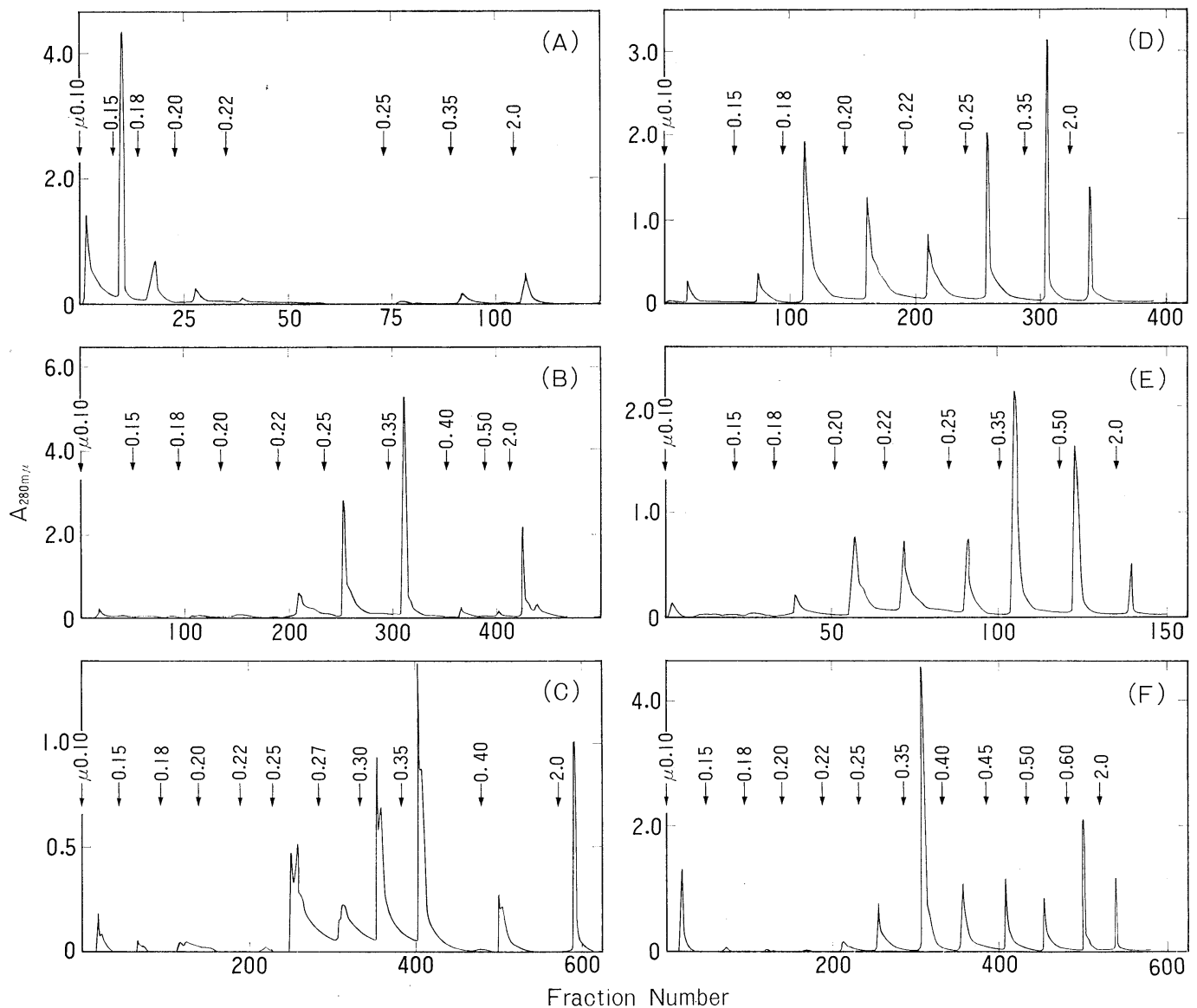

Fig. 2. DEAE-cellulose chromatography of crude preparations of thyroglobulin obtained from various animal species by salting-out. Thyroglobulins used were: (A), Xenopus laevis; (B), chicken; (C), whale; (D), hog; (E), monkey; (F) human. Although the amount of material applied to the column varied from experiment to experiment, the ratio of DEAE-cellulose to protein was kept to approximately $60: 1$ on the dry weight basis. Stepwise elution was performed as indicated by arrows. Fraction size was approximately $15 \mathrm{~m} l$.

in addition to $19 \mathrm{~S}$ thyroglobulin; increasing the ionic strength of the eluant further resulted in the gradual increase in the content of $27 \mathrm{~S}$ protein. These chromatographic characteristics of hog thyroglobulin is generally in accordance with the results previously reported (Ui et al., 1961), although the elution schedule was a little different.

In comparison to hog thyroglobulin, chromatographic behavior of thyroglobulin preparations obtained from other species were considerably different, although chromato- graphic fractionation was always observed. These thyroglobulins may be classified into two groups according to their affinities to the ion-exchanger.

In the first group, thyroglobulin was eluted at much lower ionic strengths than those needed for the elution of hog thyroglobulin. Xenopus laevis thyroglobulin belongs to this group. As seen in Figure 2A, a fairly large amount of proteins $(26 \%)$ emerged at ionic strength 0.10 during the washing of the column posterior to sample application. Ultracentri- 
fugal analysis showed that this fraction consisted of mainly thyroglobulin together with a slight amount of the slower component. At ionic strength 0.15 , approximately a half of the total protein applied was eluted; this fraction contained, in addition to $19 \mathrm{~S}$ thyroglobulin, a small amount of $27 \mathrm{~S}$ protein. On increasing the ionic strength further, no important amount of thyroglobulin was eluted.

In the second group, thyroglobulin was more strongly retained on DEAE-cellulose. Chicken (Fig. 2B), whale (Fig. 2C), monkey (Fig. 2E) and human (Fig. 2F) thyroglobulins were not eluted from the column until the ionic strength of the eluant was increased up to 0.20 or even higher. The largest amount of thyroglobulin was obtained at ionic strength 0.35 . It was also observed that chicken and whale thyroglobulins were eluted in a relatively narrow range of ionic strength $(0.25-$
0.35). In monkey and human thyroglobulins, the ionic strength had to be increased as high as 0.50 or 0.60 for the achievement of complete elution of thyroglobulin.

In the case of rat thyroglobulin, a little different elution schedule was adopted due to the limited amount of the sample available. When ionic strengths of $0.15,0.20,0.40$ and 2.0 were used successively for elution, the amounts of the protein eluted was 5, 22, 53 and $14 \%$ (of the total proteins applied), respectively. When hog thyroglobulin was eluted under the same conditions, the values obtained were 1, 45, 48 and $5 \%$, respectively. This suggests that rat thyroglobulin is retained on DEAE-cellulose more strongly than hog thyroglobulin and is to be classified in the second group.

\section{Sedimentation Coefficients}

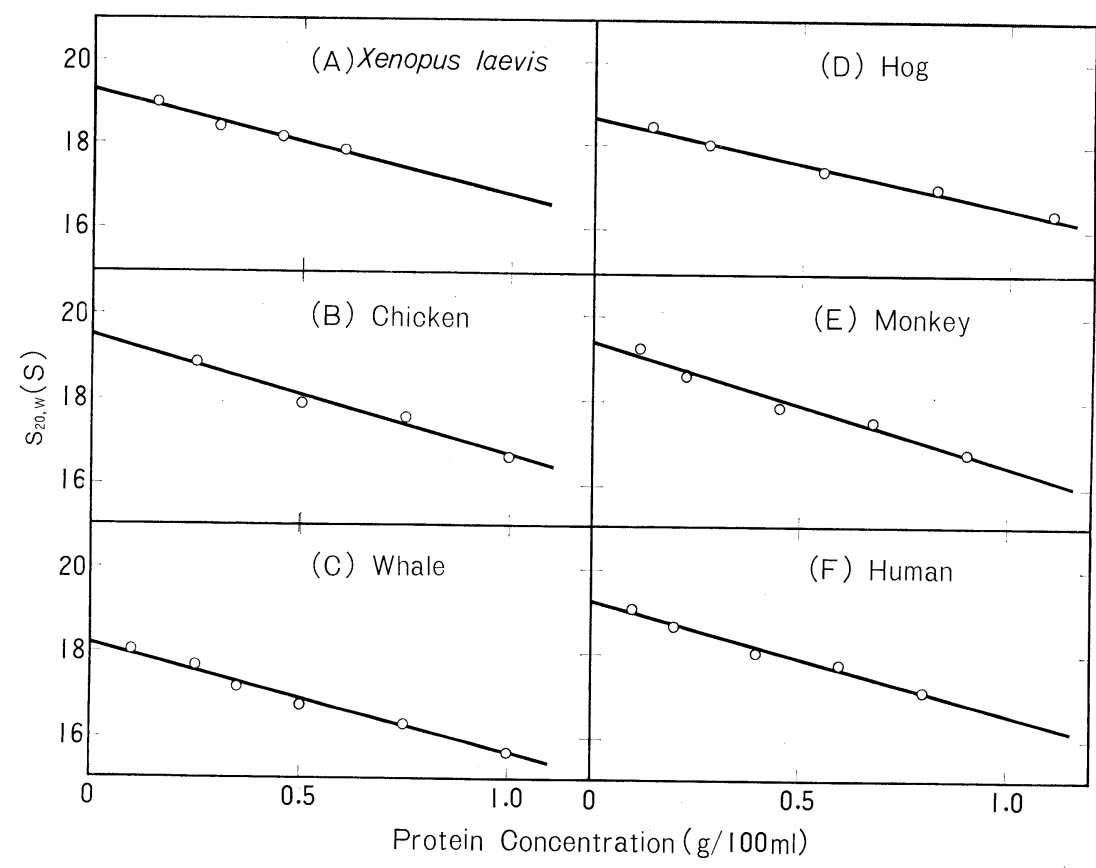

Fig. 3. Concentration dependence of the sedimentation coefficients of chromatographically purified preparations of thyroglobulin obtained from various animal species. The sources of thyroglobulin and the ionic strength employed for the elution of each preparation are: (A) Xenopus laevis, 0.10; (B) chicken, 0.35; (C), whale, 0.25; (D) hog, 0.25 ; $(\mathrm{E})$, monkey, 0.35 ; $(\mathrm{F})$, human, 0.35 . All measurements were carried out in a phosphate buffer of $\mathrm{pH} 6.5$ at ionic strength 0.2 . 
Chromatographically purified preparations of thyroglobulin obtained from various animal species were subjected to velocity ultracentrifugation at different protein concentrations. The sedimentation coefficient at infinite dilution $\left(\mathrm{s}^{\circ}{ }_{20, W}\right)$ was calculated by extrapolation of the experimental values for each preparation. These results are shown in Figuer 3.

Chicken, monkey, Xenopus laevis or human thyroglobulin showed a value of $\mathrm{S}_{20, W}$ higher than $19 \mathrm{~S}$; the values obtained were 19.5, 19.4, 19.3 and 19.2 S, respectively. The $\mathrm{s}^{\circ}{ }_{20, W}$ of hog thyroglobulin was considerably lower and showed a value of $18.6 \mathrm{~S}$ in close agreement with that reported previously (Tarutani and Ui, 1968).

The $\mathrm{S}_{20, W}$ of whale thyroglobulin was exceptionally low and a value of $18.2 \mathrm{~S}$ was obtained. Although it is now generally believed that the sedimentation coefficient of thyroglobulin is somewhat dependent upon the iodine content of the preparation and poorly- or non-iodinated thyroglobulin shows a significantly lower sedimentation coefficient than normal thyroglobulin ( $c f$. de Crombrugghe et al., 1967), the preparation of whale thyroglobulin used in the present study was not anomalous and contained $0.48 \%$ of iodine.

\section{Polyacrylamide Gel Electrophoresis and Isoelectric Focusing}

In Figure 4 are shown thin-layer polyacrylamide gel electrophoresis patterns obtained with different preparations of thyroglobulin at $\mathrm{pH}$ 8.60. In addition to the main band corresponding to $19 \mathrm{~S}$ thyroglobulin, some preparations showed the presence of one or more slowly migrating components presumably due to $27 \mathrm{~S}$ iodoprotein and other heavier components contained in these preparations.

The mobility of chicken thyroglobulin was definitely higher and that of Xenopus laevis thyroglobulin was slower than the mobilities of mammalian thyroglobulins. It was also seen that whale thyroglobulin migrated at a slightly slower rate than other mammalian

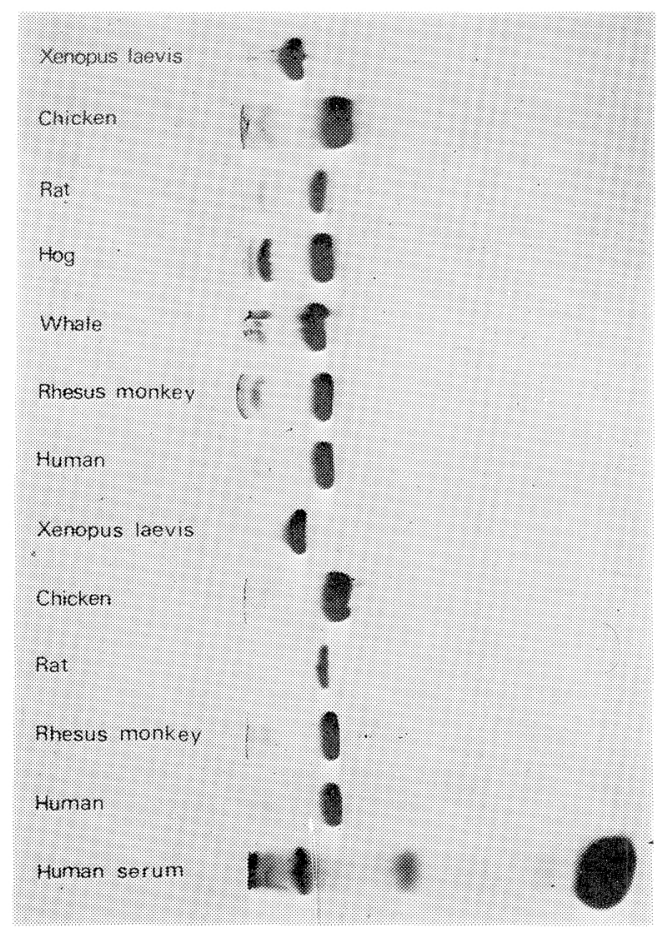

Fig. 4. Thin-layer polyacrylamide gel electrophoresis of chromatographically purified thyroglobulins from various animal species. Approximately $100 \mu \mathrm{g}$ of each preparation was applied in the slot prepared in $4 \%$ gel plate with a thickness of $1 \mathrm{~mm}$. Samples shown in this photograph are those used in sedimentation studies (see legend of Fig. 3) and rat thyroglobulin eluted at ionic strength 0.40 . Run was also made with human serum as a control. The buffer used was a veronal buffer of $\mathrm{pH} 8.60$, ionic strength 0.10 . Electrophoresis was continued at a constant current of $2 \mathrm{~mA} / \mathrm{cm}$ for $9 \mathrm{hr}$. All proteins migrated to the anode (right). Staining with amide black $10 \mathrm{~B}$.

thyroglobulins.

The isoelectric points of some preparations of thyroglobulin were determined by isoelectric focusing. As observed with hog thyroglobulin (Ui, 1971a), all the preparations examined showed a broad and complicated curve as shown in Figure 5, indicating the microheterogeneity of these preparations with respect to isoelectric point. A mean isoelectric point was calculated from the centroidal ordinate of the curve for each thyroglobulin 

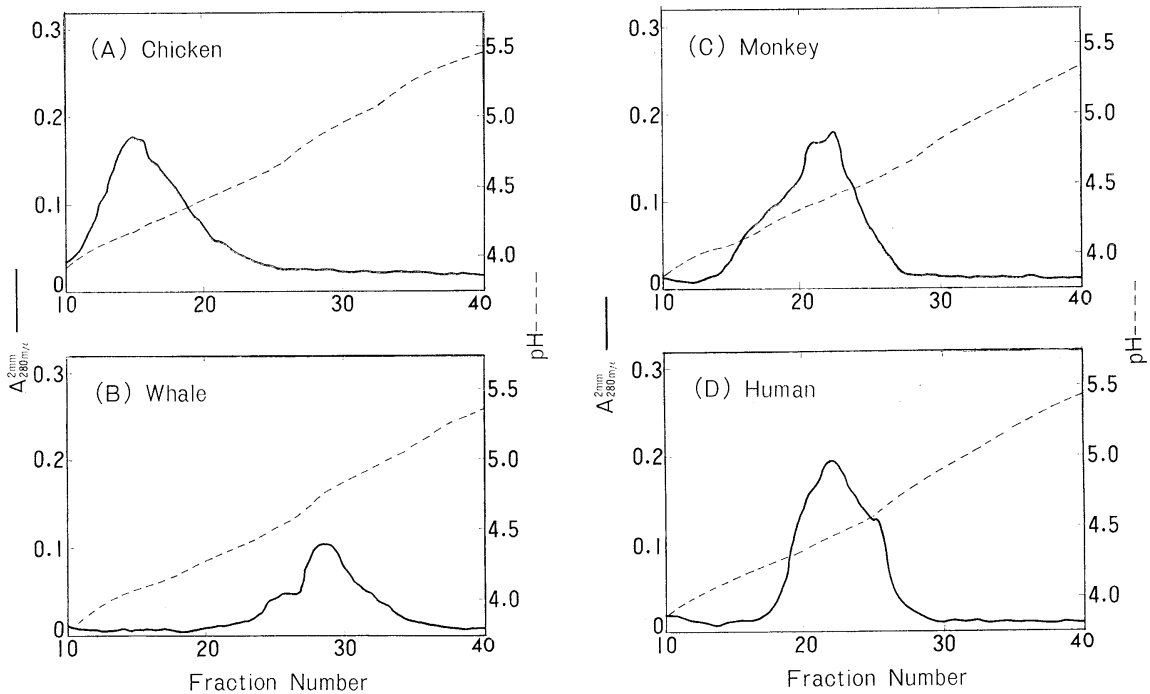

Fig. 5. Isoelectric focusing profiles of some preparations of thyroglobulin. (A), chicken; (B), whale; (C), monkey; (D), human. Approximately $9 \mathrm{mg}$ of thyroglobulin was applied for each experiment. Electrolysis was continued for $4-5$ days at $5^{\circ} \mathrm{C}$.

Table 1. Isoelectric Points of Thyroglobulins from Some Animal Species Determined by Isoelectric Focusing

\begin{tabular}{lc}
\hline \hline Species & Isoelectric point \\
\hline Chicken & 4.2 \\
Whale & 4.6 \\
Hog & $4.5^{*}$ \\
Monkey & 4.4 \\
Human & 4.4 \\
\hline
\end{tabular}

*This value was taken from the previous report (Ui, $1970 \mathrm{a}, \mathrm{b})$.

and the results are shown in Table 1.

It was found that the isoelectric point of chicken thyroglobulin was at $\mathrm{pH} 4.2$ and definitely lower than that of other thyroglobulins ( $\mathrm{pH} 4.4-\mathrm{pH} 4.6)$.

\section{Iodine Content}

Some representative results of iodine analysis are given in Table 2.

In almost all species studied, different fractions of thyroglobulin obtained by DEAEcellulose chromatography showed more or less different iodine contents, indicating the heterogeneity of thyroglobulin with respect to
Table 2. Iodine Content of the Some Selected Preparations of Thyroglobulins from Various Species

\begin{tabular}{lcc}
\hline \hline Species & $\begin{array}{c}\text { Ionic strength used } \\
\text { for elution from } \\
\text { DEAE-cellulose }\end{array}$ & $\begin{array}{c}\text { Iodine content } \\
(\%)\end{array}$ \\
\hline Xenopus laevis & 0.10 & 0.41 \\
Chicken & 0.15 & 0.61 \\
& 0.25 & 1.37 \\
Whale & 0.35 & 1.39 \\
Rat & 0.25 & 0.48 \\
& 0.35 & 0.60 \\
Hog & 0.20 & 0.29 \\
& 0.40 & 0.56 \\
& 0.18 & 0.48 \\
& 0.20 & 0.51 \\
Monkey & 0.22 & 0.58 \\
& 0.25 & 0.77 \\
Human & 0.35 & 0.72 \\
& 0.20 & 0.65 \\
& 0.35 & 0.88 \\
& 0.22 & 0.44 \\
& 0.35 & 0.52 \\
& 0.50 & 0.48 \\
\hline
\end{tabular}

the degree of iodination. The fraction eluted at a lower ionic strength generally gave a lower iodine content than the fraction eluted 
Table 3. Amino Acid Composition of Chromatographically Purified Preparations of Thyroglobulins Obtained from Various Animal Species

\begin{tabular}{lrrrrrrr}
\hline \hline \multirow{2}{*}{ Amino acids } & \multicolumn{7}{c}{ Moles per 100 moles of total amino acids } \\
\cline { 2 - 7 } & $\begin{array}{c}\text { Xenopus } \\
\text { laevis }\end{array}$ & Chicken & Whale & Rat & Hog & Monkey & Human \\
\hline Lysine & 5.0 & 3.7 & 2.9 & 3.3 & 2.5 & 3.4 & 3.4 \\
Histidine & 2.3 & 1.1 & 1.5 & 1.4 & 1.3 & 1.8 & 1.4 \\
Arginine & 4.0 & 4.2 & 5.6 & 5.1 & 6.7 & 6.0 & 5.9 \\
Aspartic acid & 10.5 & 9.6 & 7.3 & 8.7 & 7.2 & 8.1 & 8.4 \\
Threonine & 5.7 & 5.3 & 5.5 & 5.3 & 5.3 & 5.1 & 5.4 \\
Serine & 9.7 & 8.9 & 8.9 & 9.5 & 8.7 & 9.7 & 9.7 \\
Glutamic acid & 11.7 & 12.2 & 12.4 & 13.5 & 12.5 & 12.9 & 13.1 \\
Proline & 5.7 & 9.7 & 7.5 & 5.8 & 7.8 & 7.7 & 7.1 \\
Glycine & 7.3 & 7.3 & 8.2 & 7.6 & 7.7 & 7.5 & 7.7 \\
Alanine & 5.7 & 6.3 & 8.5 & 6.4 & 9.7 & 7.8 & 7.6 \\
Half cystine & 2.4 & 2.6 & 3.4 & 4.2 & 3.8 & 2.3 & 2.6 \\
Valine & 6.2 & 5.8 & 6.7 & 6.7 & 5.9 & 6.2 & 6.4 \\
Methionine & 1.8 & 1.3 & 1.2 & 1.6 & 0.9 & 1.4 & 1.4 \\
Isoleucine & 4.2 & 4.2 & 3.0 & 3.6 & 2.5 & 2.9 & 2.9 \\
Leucine & 9.3 & 9.1 & 9.9 & 9.6 & 10.3 & 9.6 & 9.5 \\
Tyrosine & 2.5 & 3.2 & 2.1 & 2.4 & 2.2 & 2.3 & 2.2 \\
Phenylalanine & 5.9 & 5.5 & 5.3 & 5.2 & 4.9 & 5.3 & 5.3 \\
\hline Total acidic & 22.2 & 21.8 & 19.7 & 22.2 & 19.7 & 21.0 & 21.5 \\
Total basic & 11.3 & 9.0 & 10.0 & 9.8 & 10.5 & 11.2 & 10.7 \\
\hline
\end{tabular}

later. This is in harmony with the previous finding with hog, bovine or human thyroglobulin (Ui et al., 1961; Robbins, 1963; Valenta et al., 1968).

Exceptionally, two major fractions of chicken thyroglobulin showed essentially the same iodine content. It is likely that chicken thyroglobulin is more homogeneous than other thyroglobulins at least as far as the present chicken preparation is concerned. The relatively simple elution pattern of chicken thyroglobulin obtained on DEAE-cellulose chromatography (see Fig. 2B) might consist with this view.

It is also to be noted that chicken thyroglobulin showed an unusually high iodine content (mean value: $1.38 \%$ ). This value is much higher than the values hitherto reported for mammalian thyroglobulins; there has been no report on the iodine content of thyroglobulins obtained from non-mammalian vertebrates.

Xenopus laevis thyroglobulin as well as any mammalian thyroglobulin studied was not unusual with respect to the content of iodine.

\section{Amino Acid Composition}

Amino acid analyses were carried out on the chromatographically purified major fractions obtained from various thyroglobulins. The results expressed as moles per 100 moles of total amino acids recovered are given in Table 3. Iodo-amino acids, tryptophan and amide were not determined. Values for tyrosine would contain a portion of iodo-amino acids which were deiodinated during the hydrolysis.

Generally speaking, all the preparations were rather similar in amino acid composition in spite of a large variety of animal species used. However, non-mammalian thyroglobulins showed considerably large differences from mammalian thyroglobulins in the contents of some amino acids. When the sum of acidic amino acids or of basic amino acids was compared, chicken thyroglobulin gave the 
lowest value for the total basic amino acids and the highest value for the molar ratio of the total acidic to the total basic amino acids. Xenopus laevis thyroglobulin, on the other hand, showed a relatively high value for the total content of basic amino acids. It was also found that the amounts of aspartic acid and glutamic acid residues were rather similar in Xenopus laevis thyroglobulin, while all other thyroglobulins, especially mammalian thyroglobulins, were much more abundant in glutamic acid than aspartic acid.

So far there has been no report on the amino acid composition of non-mammalian thyroglobulin except that of duck thyroglobulin by Rolland et al. (1966). It is of interest that our result on chicken thyroglobulin was very similar to that of duck thyroglobulin. The amino acid compositions of rat, hog and human thyroglobulins obtained in this investigation were fairly in close agreement with those reported by other investigators (Rolland et al., 1966; Pierce et al., 1965).

\section{Immunodiffusion}

Double diffusion tests were made with chromatographically purified preparations of thyroglobulin obtained from various species in order to examine species specificity of thyroglobulin. Antisera used were prepared by immunizing rabbits with chromatographically purified preparations of chicken, whale, hog and human thyroglobulins. Some of the representative immunodiffusion patterns obtained are shown in Figure 6, and the results are summarized in Table 4 . One precipitin line was always observed when these antisera were reacted with thyroglobulin obtained from the same species as used for the preparation of antisera.

It was shown that two non-mammalian thyroglobulins were entirely unrelated to mammalian thyroglobulins. Xenopus laevis thyroglobulin did not give a precipitin line when reacted with all anti-mammalian thyroglobulin antisera tested. This thyroglobulin
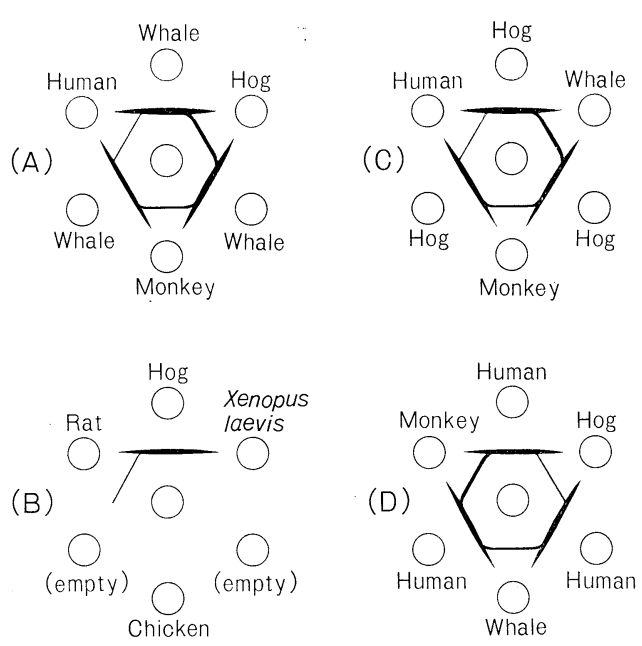

Fig. 6. Double diffusion in agar gel of various preparations of thyroglobulin. Central wells contained: (A), anti-whale thyroglobulin antiserum; (B) and (C), anti-hog thyroglobulin antiserum; (D), anti-human thyroglobulin antiserum. In peripheral wells, purified preparations of thyroglobulin were placed as shown in the figure.

was also unreactive with anti-chicken thyroglobulin antiserum. There was also a complete absence of cross-reaction between chicken thyroglobulin and rabbit antisera to mammalian thyroglobulins, or between mammalian thyroglobulins and antiserum to chicken thyroglobulin. This is in agreement with the results of other investigators obtained by precipitin analysis using less purified preparations of thyroglobulin as antigen (Hecktoen et al., 1927; Stokinger and Heidelberger, 1937).

Mammalian thyroglobulins were often found to be more or less related immunochemically to each other (see Table 4). This result is in harmony with the results of the classical work done by above-mentioned investigators and more recent investigation by Rose and Witebsky (1955) using different techniques. In immunodiffusion, rat thyroglobulin showed a weak band only against anti-hog thyroglobulin antiserum. Whale thyroglobulin reacted not only with anti-whale 
Table 4. Cross Reaction of Thyroglobulins from Various Animal Species

\begin{tabular}{lcccccccc}
\hline \hline \multicolumn{7}{c}{ Antisera } & \multicolumn{7}{c}{ Source of thyroglobulin used as antigen } \\
\cline { 2 - 7 } & $\begin{array}{c}\text { Xenopus } \\
\text { laevis }\end{array}$ & Chicken & Whale & Rat & Hog & Monkey & Human \\
\hline Anti-chicken & - & ++ & - & - & - & - & - \\
Anti-whale & - & - & ++ & - & + & + & $(+)$ \\
Anti-hog & - & - & + & $(+)$ & ++ & + & $(+)$ & ++ \\
Anti-human & - & - & $(+)$ & - & $(+)$ & + & +
\end{tabular}

*The following symbols are used: ++ , strong reaction; + , cross reaction; $(+)$, weak cross reaction; - , no reaction.

thyroglobulin antiserum, but also with antisera to hog thyroglobulin and less significantly to human thyroglobulin; in the latter two cases, a weak line fused as a spur with the whale line. The cross reaction between hog thyroglobulin and antisera against whale and human thyroglobulins was also observed, although the latter cross reaction was much weaker than the former reaction. Monkey and human thyroglobulins similarly cross-reacted with anti-whale and anti-hog antisera. The cross reaction between monkey thyroglobulin and anti-human thyroglobulin antiserum was considerably strong; the line formed did not fuse completely with the human line, but produced a spur.

\section{Discussion}

Comparison of the properties of thyroglobulins obtained from various species has to be made with caution, since thyroglobulin is known to consist of a variety of molecules which differ in iodine content and some other properties ( $c f$., Rall et al., 1964; Robbins et al., 1966; Tarutani and Ui, 1969 a,b). In the present investigation, a large number of thyroid glands were used for preparing each thyroglobulin preparation in order to reduce the possible variations among individuals as far as possible, although only three glands were available in the case of whales. It is also to be emphasized that all the preparations of thyroglobulin were purified and fractionated by DEAE-cellulose chromatography and the major components obtained were chiefly used for characterization.

The results of studies presented in this paper indicate that all thyroglobulins so far examined are rather remarkably similar in spite of a great variety of species ranging from amphibia to primates. However, closer inspection of the data has revealed the presence of various kinds of species specificity, especially between non-mammalian and mammalian thyroglobulins. These variations between species appeared even greater than in the case of other proteins like insulin and cytochrome c.

Xenopus laevis thyroglobulin differed most distinctly from other thyroglobulins. It was much more soluble in concentrated solutions of ammonium sulfate and eluted from DEAEcellulose at definitely lower ionic strengths than in the case of other thyroglobulins. The lower electrophoretic mobility to the anode at $\mathrm{pH} 8.6$ is presumably ascribed to the higher contents of basic amino acids. Immunologically, Xenopus laevis thyroglobulin seemed to be completely unrelated to thyroglobulins from other classes of vertebrates. Interestingly enough, however, this thyroglobulin showed approximately the same sedimentation coefficient as most other thyroglobulins and its iodine content was also not unusual.

Chicken thyroglobulin, which was also immunologically unrelated to other classes of thyroglobulin, has been found to be an exceptionally acidic thyroglobulin with the isoelectric point at $\mathrm{pH} 4.2$. This is in harmony 
with the result of amino acid analysis and the finding that it moved fastest in electrophoresis at $\mathrm{pH}$ 8.6. The markedly high iodine content and relatively high degree of homogeneity (on DEAE-cellulose chromatography) observed with chicken thyroglobulin are worth notice. However, it remains to be examined whether these are general tendencies of this class of thyroglobulin. The sedimentation coefficient of chicken thyroglobulin was very close to that of most other thyroglobulins.

Mammalian thyroglobulins were generally very similar and immunological cross reactivity was often observed in double diffusion when mammalian thyroglobulins were examined with antisera to some mammalian thyroglobulins. Whale thyroglobulin was special in that its sedimentation coefficient $\left(\mathrm{s}^{\circ}{ }_{20, W}\right), 18.2 \mathrm{~S}$, was much lower than the value for most other thyroglobulins which showed $\mathrm{S}^{\circ}{ }_{20, W}$ slightly higher than $19 \mathrm{~S}$. Hog thyroglobulin gave an intermediate value of $18.6 \mathrm{~S}$. These variations in sedimentation coefficient deserve further investigation, since systematic studies on the sedimentation coefficients of various thyroglobulins have not been made.

In addition to species variation as well as similarity in the properties among various thyroglobulins, the present investigation also provided some information concerning thyroglobulin-related proteins in the thyroid glands of various animal species. Although the presence of $12 \mathrm{~S}$ component, which is considered to be a stable subunit of thyroglobulin, has been reported in the thyroid glands of some lower vertebrates and guinea pig (Roche et al., 1968), all the animals used in the present investigation including amphibia and aves did not contain this component. The presence of $27 \mathrm{~S}$ iodoprotein, likely the dimer molecule of thyroglobulin, was always observed in all animals in agreement with the results of previous investigators (Roche et al., 1968).

It is surprising that a similar, comparative study on the properties of thyroglobulins from various animal species has not been hitherto attempted. The present investigation appears to indicate that this type of approach is rewarding. It is hoped that extensive investigations in future of species variations in thyroglobulin will enlarge our understanding of the nature of thyroglobulin and supply information about phylogenetic relationships.

\section{Acknowledgements}

The authors wish to express their thanks to Dr. O. Tarutani for his help and invaluable advice. The skilful assistance of Mr. T. Kondo in various phases of this investigation is greatly appreciated.

\section{References}

de Crombrugghe, B., H. Edelhoch, C. Beckers and M. de Visscher (1967). J. Biol. Chem. 242, 5681 .

Gorbman, A. and H. A. Bern. A Text Book of Comparative Endocrinology. John Wiley \& Sons, New York, p. 99 (1962).

Gross, W. G., L. K. Wood and J. S. McHargue (1948). Anal. Chem. 20, 900.

Hektoen, L., H. Fox and K. Schulhof (1927). J. Infect. Dis. 40, 641.

Lachiver, F., Y. A. Fontaine and A. Martin. In "Current Topics in Thyroid Research" (C. Cassano and M. Andreoli, eds.), Academic Press, New York, p. 182 (1965).

Lachiver, F., A. Martin and M. Fontaine (1966). Gunma Symp. Endocrinol. 3, 109.

Ogita, Z. (1965). Metabolism and Disease 2, 331 (in Japanese).

Ouchterlony, Ö. In "Progress in Allergy" (P. Kallós and B. H. Waksman, eds.), Vol. 6, S. Karger, Basel, p. 30 (1962).

Pierce, J. G., A. B. Rawitch, D. M. Brown and P. G. Stanley (1965). Biochim. Biophys. Acta 111, 247.

Rall, J. E., J. Robbins and C. G. Lewallen. In "The Hormones" (G. Pincus, K. V. Thimann and E. B. Astwood, eds.), Vol. V, Academic Press, New York, p. 159 (1964).

Robbins, J. (1963). J. Biol. Chem. 238, 182. 
Robbins, J., G. Salvatore, G. Vecchio and N. Ui (1966). Biochim. Biophys. Acta 127, 101. Roche, J., G. Salvatore, L. Sena, S. Aloj and I. Covelli (1968). Comp. Biochem. Physiol. 27, 67.

Rolland, M., J. Bismuth, J. Fondarai and S. Lissitzky (1966). Acta Endocrinol. 53, 286.

Rose, N. R. and E. Witebsky (1955). J. Immunol. 75, 282.

Salvatore, G., G. Vecchio, M. Salvatore, H. J. Cahnmann and J. Robbins (1965). J. Biol. Chem. 240, 2935.

Schneider, A. B., H. Bornet and H. Edelhoch (1970). Ibid. 245, 2672.

Shulman, S. and J. P. Armenia (1963). Ibid. 238, 2723.

Spackman, D. H., W. H. Stein and S. Moore (1958). Anal. Biochem. 30, 1190.

Spiro, R. G. and M. J. Spiro (1965). J. Biol. Chem. 240, 997.

Stokinger, H. E. and M. Heidelberger (1937).
J. Exptl. Med. 66, 251.

Svensson, H. (1962). Arch. Biochem. Biophys. Suppl. 1, 132.

Tarutani, O. and N. Ui (1968). Biochem. Biophys. Res. Commun. 33, 733.

Tarutani, O. and N. Ui (1969a). Biochim. Biophys. Acta 181, 116.

Tarutani, O. and N. Ui (1969b). Ibid. 181, 136.

Ui, N. Proc. 6th Intern. Thyroid Conf., Vienna, 1970, in press (1971a).

Ui, N. (1971b). Biochim. Biophys. Acta, in press.

Ui, N. and O. Tarutani (1961). J. Biochem. 50, 508.

Ui, N., O. Tarutani, Y. Kondo and H. Tamura (1961). Nature 191, 1199.

Valenta, L., M. Roques, J. Torresani, M. Rolland and S. Lissitzky (1968). Biochim. Biophys. Acta 168, 507.

Vesterberg, O. and H. Svensson (1966). Acta Chem. Scand. 20, 820. 